\title{
Estudo laboratorial do uso de cefovecina em cães sadios
}

\section{Laboratory evaluation of the use of cefovecin in healthy dogs}

\author{
Cecília Braga Laposy ${ }^{1 *}$; Dheywid Karlos Mattos Silva²; Júlia Neves Pessoto³
}

\section{Resumo}

A cefovecina é a mais nova cefalosporina semissintética de terceira geração, indicada para tratamento de infecções de pele e do trato urinário de cães e gatos. O principal diferencial da cefovecina para as outras cefalosporinas é sua alta ligação às proteínas plasmáticas, o que lhe permite longo período de ação. Devido à ausência de trabalhos demonstrando alterações laboratoriais provocadas pelo uso da cefovecina, este trabalho teve o objetivo de avaliar a influência da terapia com cefovecina sobre as proteínas séricas e suas frações, valores do hemograma, atividade de uréia e creatinina, hemogasometria, distribuição do diâmetro das plaquetas (PDW) e contagem plaquetária em cães hígidos. Foram usados dez cães adultos (machos $\mathrm{n}=4$; fêmeas $\mathrm{n}=6$ ), com peso médio de 10,03 $\pm 3,54 \mathrm{Kg}$, incluídos nos experimento mediante exames clínicos e laboratoriais. Os cães receberam por via subcutânea a cefovecina sódica $\left(\right.$ Convenia $\left.{ }^{\circledR}\right)$, na dose de $8 \mathrm{mg} / \mathrm{Kg}$ e foram acompanhados clínica e laboratorialmente nos dias 1, 2, 3, 7, 14 e 21 após o tratamento (dpt). Apesar de os valores permanecerem dentro da faixa de normalidade para a espécie, houve uma diminuição significativa $(\mathrm{P}<0,05)$ nos valores da proteína sérica, globulinas, hemoglobina e volume globular aos 21(dpt). O diâmetro das plaquetas (PDW), bem como sua contagem diminuíram aos 7 e 21 dias, mas sem diferença significativa $(P>0,05)$, respectivamente. Não houve alterações nas atividades de uréia e creatinina, hemogasometria e valores leucocitários após a administração da cefovecina. Conclui-se que a cefovecina pode ser administrada em cães saudáveis sem causar alterações laboratoriais significantes.

Palavras-chave: Cães, cefovecina, hematologia, plaquetas

\begin{abstract}
Cefovecin is the third generation semi synthetic cephalosporin. The drug is indicated for treatment of skin and urinary infections. The main difference between cefovecin and the others cephalosporins is the highest plasma protein binding, providing an extend action. Due to lack of laboratory studies demonstrating changes caused by the use of cefovecin, the aim of this study was to evaluate the influence of cefovecin therapy on the serum proteins, hemogram, urea and creatinine activities, blood gas, platelet distribution width (PDW) and platelet count. Ten healthy dogs (males $n=4$; females $n=6$ ), average weight of $10.03 \pm 3.54 \mathrm{~kg}$ were used. The animals were kept in individual cages all over the experiment. The animals were submitted to the clinical and laboratory examinations as an inclusion criterion for the experiment. Sodium cefovecin $\left(\right.$ Convenia $\left.^{\circledR}\right)$ was subcutaneously administered $(8 \mathrm{mg} /$ $\mathrm{kg}$ ) to the dogs. Clinical evaluation and laboratorial analysis was performed prior and at 1, 2, 3, 7, 14 and 21 days post treatment (dpt). Despite to be in accordance with the reference values, it was observed a statistically significant $(\mathrm{p}<0.05)$ decrease in total serum protein, globulin, hemoglobin and packed cell volume at $21 \mathrm{dpt}$. The platelet distribution width (PDW) and platelet count were increased at seven and 21 days ( $>0.05)$, respectively. No changes in urea and creatinine activities, blood gas and leukocyte values were observed in the dogs after the administration of cefovecin. In conclusion, cefovecin can be administered in healthy dogs without causing significant laboratory abnormalities.
\end{abstract}

Key words: Dogs, cefovecin, hematology, platelet

\footnotetext{
Prof $^{a} \mathrm{Dr}^{\mathrm{a}}$ da Universidade do Oeste Paulista, UNOESTE, Presidente Prudente, SP. E-mail: claposy@unoeste.br

2 Discente de Mestrado Ciência Animal, UNOESTE, Presidente Prudente, SP. E-mail:dheywid@hotmail.com

3 Discente de Medicina Veterinária, UNOESTE, Presidente Prudente, SP. E-mail:juliajnp@ig.com.br

Autor para correspondência
} 
A rotina dermatológica é uma das mais frequentes na clínica de pequenos animais. Levantamentos realizados demonstram que as dermatopatias, tanto primárias quanto secundárias, representam mais que $31 \%$ do atendimento clínico (CARDOSO et al., 2011). Além das infecções cutâneas, as doenças do trato urinário vêm logo em seguida na casuística clínica, acometendo cerca de $30 \%$ dos cães e gatos (ISHII; FREITAS; ARIAS, 2011).

As cefalosporinas são classificadas em "gerações", segundo características e ordem cronológica de sua síntese. Atualmente, existem quatro gerações dessa família de antibióticos. A cefovecina sódica é um antibiótico de terceira geração, recomendado para cães e gatos, aplicado pela via subcutânea em dose única de $8 \mathrm{mg} / \mathrm{kg}$. Seu diferencial em relação às outras cefalosporinas e demais classes de antibióticos, é sua alta ligação às proteínas plasmáticas, o que lhe confere longo período de ação e, consequentemente, reduzido número de aplicações. Em alguns casos, o fármaco pode ser repetido 14 dias após a primeira aplicação (STEGEMANN; SHERINGTON, J.; BLANCHFLOWER, 2006; STEGEMANN et al., 2006b).

O uso da cefovecina em medicina veterinária vem se ampliando, uma vez que este antibiótico possibilita maior adesão ao tratamento pelo proprietário, reduz a incidência de resistência antimicrobiana e ainda minimiza o estresse causado por uma terapia prolongada (STEGEMANN et al., 2006a, 2006b). Estas características têm sido apontadas pelos médicos veterinários como as grandes vantagens para o seu uso, tanto em animais de companhia (STEGEMANN et al., 2006a) como os criados em cativeiro (PAPP et al., 2010).

Devido à escassez de trabalhos que demonstram os efeitos colaterais provocados pela cefovecina, esta pesquisa teve por objetivo verificar possíveis alterações renais, hematológicas, hemogasométricas e plaquetárias provocadas pelo uso deste fármaco em cães saudáveis higidos.
Foram utilizados dez cães adultos, provenientes do canil da Universidade do Oeste Paulista, sem raça definida, pesando em média $10,03 \mathrm{Kg} \pm 3,54$, sendo 4 machos e 6 fêmeas. Adotaram-se como critérios de inclusão, a realização de exame físico, hemograma com pesquisa de hematozoários, dosagens de uréia e creatinina séricas e hemogasometria, para avaliação da higidez dos animais. Os cães permaneceram durante todo o experimento em baias individuais, recebendo ração comercial duas vezes ao dia e água ad libitum. Após a avaliação inicial, os animais receberam cefovecina sódica (Convenia, Laboratórios Pfizer-LTDA), na dose única de $8 \mathrm{mg} / \mathrm{Kg}$ por via subcutânea e foram avaliados clinicamente uma vez ao dia sempre antes da coleta sanguínea e laboratorialmente durante 21 dias. As coletas foram realizadas em sete momentos: M0 (basal), M1 (24h), M2 (48h), M3 (72h), M4 (7 dias), M5 (14 dias) e M6 (21 dias). Para a realização da hemogasometria para análise de $\mathrm{pH}, \mathrm{pCO}_{2}, \mathrm{pO}_{2}$ e $\mathrm{HCO}_{3}^{-}$(Hemogasômetro Cobas, Roche Diagnóstica-Brasil S.A.), amostras de sangue venoso foram colhidas da veia cefálica e acondicionadas em seringas plásticas de $1 \mathrm{~mL}$ contendo $0,1 \mathrm{~mL}$ de heparina sódica. Para a dosagem de uréia, creatinina, proteína sérica total (PST) e albumina, foram coletados pela veia jugular, $5 \mathrm{~mL}$ de sangue total que foram centrifugados a $3.500 \mathrm{x}$ g/ $5 \mathrm{~min}$ (3.500 rpm (1944G) durante 5 minutos) o soro foi acondicionado em tubos plásticos e mantidos congelados a $-20^{\circ} \mathrm{C}$, até o momento do processamento. As análises bioquímicas foram realizadas em aparelho semi-automático (Quick Lab II, Drake Eletrônica e Comércio- LTDA), utilizando-se kits de reagentes específicos (Labtest Diagnóstica S.A.). Para o hemograma, contagem e determinação do diâmetro das plaquetas (PDW), outros $5 \mathrm{~mL}$ de sangue foram colhidos da veia jugular e colocados em frascos contendo anticoagulante EDTA a 10\% na proporção de 1 gota para $3 \mathrm{~mL}$ de sangue. Os exames foram processados em analisador hematológico (POCH-100IV DIFF, Sysmex do Brasil Indústria e Comércio- LTDA). 
Os dados foram avaliados pela análise de variância de medidas repetidas. Posteriormente, aqueles valores que passaram pelas premissas deste exame foram submetidos ao teste de Tukey, com grau de significância de 5\%, utilizando o programa GraphPad InStat (AYRES et al., 2007).

Todos os parâmetros laboratoriais permaneceram dentro da faixa de normalidade em relação às médias obtidas no momento basal (M0). Houve uma diminuição significativa dos valores séricos de PST e globulinas verificada aos 21 dias da aplicação do fármaco. Já os valores médios de albumina oscilaram durante todo o experimento e alcançaram os níveis mais baixos com 48 horas
$(\mathrm{P}<0,05)$. A relação albumina/globulinas $(\mathrm{A} / \mathrm{G})$ não sofreu mudanças significativas com a administração da cefovecina (Tabela 1). Apesar da cefovecina possuir alta ligação (98\%) às proteínas plasmáticas, é eliminada de forma inalterada pelos rins, sem sofrer qualquer metabolização hepática. Somente a droga livre é eliminada, fato que contribui para a sua longa ação (WERNICK; MÜNTENER, 2010). O fármaco provocou a redução das proteínas séricas totais, das globulinas e albumina como já verificado com outras cefalosporinas de segunda e terceira geração (ANDRADE; GIUFFRIDA, 2008), mas apesar da diminuição, os animais não apresentaram hipoproteinemia.

Tabela 1. Valores médios \pm desvios-padrões de proteína sérica total-PST $(\mathrm{g} / \mathrm{dL})$, albumina $(\mathrm{g} / \mathrm{dL})$, globulinas $(\mathrm{g} /$ $\mathrm{dL})$, relação albumina/globulinas $(\mathrm{A} / \mathrm{G})$, uréia $(\mathrm{mg} / \mathrm{dL})$ e creatinina $(\mathrm{mg} / \mathrm{dL})$ de 10 cães submetidos a terapia com cefovecina durante 21 dias. Intervalo fisiológico segundo Kaneko, Harvey e Bruss (2008).

\begin{tabular}{lcccccc}
\hline Momentos & PST & Globulinas & Albumina & A/G & Uréia & Creatinina \\
\hline M0 (basal) & $8,6 \pm 1,6$ & $6,1 \pm 1,2$ & $2,5 \pm 0,6$ & 0,41 & $31,9 \pm 5,7$ & $0,9 \pm 0,1$ \\
M1(24 h) & $8,0 \pm 1,4$ & $5,5 \pm 1,6$ & $2,5 \pm 0,5$ & 0,45 & $28,8 \pm 6,3$ & $0,9 \pm 0,1$ \\
M2 (48h) & $7,7 \pm 0,3^{*}$ & $5,3 \pm 1,5$ & $2,4 \pm 0,4^{* *}$ & 0,45 & $30,3 \pm 5,1$ & $1,0 \pm 0,2$ \\
M3 (72h) & $7,8 \pm 1,4^{*}$ & $5,2 \pm 1,8^{*}$ & $2,6 \pm 0,5$ & 0,50 & $32,2 \pm 5,5$ & $1,0 \pm 0,3$ \\
M4 (7d) & $7,8 \pm 1,3^{*}$ & $5,2 \pm 1,6^{*}$ & $2,6 \pm 0,5$ & 0,50 & $28,7 \pm 7,5$ & $1,0 \pm 0,2$ \\
M5 (14d) & $7,8 \pm 1,3^{*}$ & $5,1 \pm 1,7^{*}$ & $2,8 \pm 0,6$ & 0,54 & $30,4 \pm 4,7$ & $1,0 \pm 0,2$ \\
M6 (21d) & $7,4 \pm 1,3^{*}$ & $4,9 \pm 1,6^{*}$ & $2,6 \pm 0,6$ & 0,53 & $30,4 \pm 6,4$ & $1,0 \pm 0,2$ \\
Valor normal & $5,4-8,0$ & $2,3-5,2$ & $2,1-4,3$ & $0,5-1,7$ & $21-60$ & $0,5-1,5$ \\
\hline
\end{tabular}

*diferença significativa em relação ao $\mathrm{M} 0(\mathrm{P}<0,05)$.

** diferença significativa em relação ao M5 $(\mathrm{P}<0,05)$.

Fonte: Elaboração dos autores.

A maioria das cefalosporinas é bem tolerada, porém há relatos de anemias causadas por múltiplos mecanismos em cães, incluindo anemia hemolítica imunomediada e depressão medular (GOSSETT, 2000). Cães que apresentam baixa quantidade de potássio eritrocitário são mais suscetíveis a desenvolver esta forma de anemia. Já a depressão medular, embora previsível, é limitante e reversível (VADEN; RIVIERE, 2003). No presente estudo, pode-se verificar uma diminuição dos valores de hemoglobina e volume globular nas primeiras 48 horas da aplicação, quando confrontados aos valores obtidos aos 21 dias, mas sem causar anemias (Tabela 2).
As atividades de uréia e creatinina não diferiram significativamente, permanecendo dentro dos valores normais durante o período avaliado. Assim como outros antibióticos $\beta$-lactâmicos, a cefovecina é eliminada primariamente pela via renal (STEGEMANN; SHERINGTON; BLANCHFLOWER, 2006). Para apresentar efeito tóxico sobre o túbulo proximal, seria necessário administrar 100 vezes a dose recomendada a fim de produzir nefropatia. O potencial nefrotóxico das cefalosporinas é incrementado quando associada aos aminoglicosídeos (VADEN; RIVIERE, 2003). 
Tabela 2. Valores médios \pm desvios-padrões de hemácias $\left(\mathrm{x} 10^{6}\right)$, hemoglobina $(\mathrm{g} / \mathrm{dL})$, volume globular - VG $(\%)$, volume corpuscular médio - VCM (fl) e concentração de hemoglobina corpuscular média - CHCM (\%) de 10 cães submetidos a terapia com cefovecina durante 21 dias. Intervalo fisiológico segundo Jain (1993).

\begin{tabular}{lccccc}
\hline Momentos & Hemácias & Hb & VG & VCM & CHCM \\
\hline M0 (basal) & $7,1 \pm 1,3$ & $14,7 \pm 2,0^{*}$ & $44,6 \pm 6,1^{*}$ & $63,2 \pm 5,6$ & $33,0 \pm 1,4$ \\
M1(24 h) & $6,9 \pm 2,2$ & $14,5 \pm 2,1^{*}$ & $43,7 \pm 6,0^{*}$ & $63,3 \pm 5,5$ & $33,5 \pm 1,5$ \\
M2 (48h) & $6,9 \pm 1,3$ & $14,4 \pm 2,1^{*}$ & $43,0 \pm 6,2^{*}$ & $62,2 \pm 5,6$ & $33,6 \pm 1,6$ \\
M3 (72h) & $6,8 \pm 1,2$ & $14,2 \pm 1,8$ & $42,2 \pm 5,3$ & $62,2 \pm 5,5$ & $33,4 \pm 1,3$ \\
M4 (7d) & $6,8 \pm 1,2$ & $14,2 \pm 2,2$ & $42,1 \pm 6,3^{*}$ & $62,1 \pm 5,4$ & $33,7 \pm 1,2$ \\
M5 (14d) & $6,9 \pm 1,3$ & $14,6 \pm 2,6^{*}$ & $43,3 \pm 7,3$ & $62,6 \pm 5,0$ & $33,7 \pm 1,2$ \\
M6 (21d) & $6,4 \pm 1,3$ & $13,6 \pm 2,7$ & $39,9 \pm 7,7$ & $62,7 \pm 4,6$ & $33,9 \pm 1,0$ \\
Valor normal & $5,5-8,5$ & $12,0-18,0$ & $37,0-55,0$ & $60,0-77,0$ & $31,0-36,0$ \\
\hline
\end{tabular}

*diferença significativa em relação a M6 $(\mathrm{P}<0,05)$.

Fonte:Elaboração dos autores.

As variáveis hemogasométricas de $\mathrm{pH}, \mathrm{pCO}_{2}$, $\mathrm{pO}_{2}$ e $\mathrm{HCO}_{3}^{-}$permaneceram sem alterações significativas ao longo do tempo $(\mathrm{P}>0,05)$. Maden et al. (2001), ao investigarem os possíveis efeitos colaterais da administração de cefquinome em cães saudáveis (cefalosporina de quarta geração) sobre os parâmetros hemogasométricos, verificaram alterações significativas nestas mesmas variáveis. Traş et al. (2001), também observaram modificações na hemogasometria de cães submetidos à terapia com enrofloxacina, mas atribuíram estas alterações à problemas respiratórios prévios dos animais.

A cefovecina, neste estudo, não ocasionou alterações significativas na quantidade e no diâmetro das plaquetas. Durante todo o período experimental, os animais não apresentaram hemorragias ou alterações sugestivas de comprometimento das funções plaquetárias.

Sendo assim, pode-se concluir que a cefovecina em dose única pode ser administrada em cães hígidos sem ocasionar importantes alterações laboratoriais. Porém, novos estudos são necessários para avaliar o efeito da droga em cães anêmicos, apresentando enteropatias, hemorragias, queimaduras graves ou insuficiência hepática.

O trabalho foi aprovado pela Comissão de Ética no Uso de Animais (CEUA) da instituição onde o ensaio foi conduzido, sob o protocolo número $702 / 2011$.

\section{Referências}

ANDRADE, S. F.; GIUFFRIDA, R. Quimioterápicos antimicrobianos e antibióticos. In.: ANDRADE, S. F. Manual de terapêutica veterinária. São Paulo: Roca, 2008. p. 38-39.

AYRES, M.; AYRES JUNIOR, M.; AYRES, D. L.; SANTOS, A. S. BIOESTAT - aplicações estatísticas nas áreas das ciências biomédicas. Ong Mamiraua, Belém, 2007. $364 \mathrm{p}$.

CARDOSO, M. J. L.; MACHADO, L. H. A.; MELUSSI, M.; ZAMARIAN, T. P.; CARNIELLI, C. M.; FERREIRA JUNIOR, J. C. M. Dermatopatias em cães: revisão de 257 casos. Archives of Veterinary Science, Curitiba, v. 16, n. 2, p. 66-74, 2011.

GOSSETT, K. A. Anemias associated with drugs and chemicals. In.: FELDMAN, B. F.; ZINKL, J. G.; JAIN, N. (Ed.). Schalm's veterinary hematology. Baltimore: Lippincott Williams \& Wilkins, 2000. p. 185-189.

ISHII, J. B.; FREITAS, J. C.; ARIAS, M. V. B. Resistência de bactérias isoladas de cães e gatos no Hospital Veterinário da Universidade Estadual de Londrina (20082009). Pesquisa Veterinária Brasileira, Rio de Janeiro, v. 31, n. 6, p. 533-537, 2011.

JAIN, N. C. Essentials of veterinary hematology. Philadelphia: Lea \& Febiger, 1993. 417 p.

KANEKO, J. J.; HARVEY, J. W.; BRUSS, M. L. Clinical biochemistry of domestic animals. San Diego: Academic Press, 2008. 916 p.

MADEN, M.; TRAŞ, B.; BAŞ, A. L.; ELMAS, M.; YAZAR, E.; BIRDANE, F. M. Investigation of biochemical and haematological side-effects of cefquinome in healthy dogs. The Veterinary Quarterly, Abingdon, v. 23, n. 1, p. 32-34, 2001. 
PAPP, R.; POPOVIC, A.; KELLY, N.; TSCHIRRETGUTH, R. Pharmacokinetics of cefovecin in squirred monkey (Saimiri sciureus), rhesus macaques (Macaca mulatta), and cynomolgus macaques (Macaca fascicularis). Journal of the American Association for Laboratory Animal Science, Memphis, v. 49, n. 6, p. 805808, 2010.

STEGEMANN, M. R.; PASSMORE, C. A.; SHERINGTON, J.; LINDEMAN, C. J.; PAPP, G.; WEIGEL, D. J.; SKOGERBOE, T. L. Antimicrobial activity and spectrum of cefovecin, a new extendedspectrum cephalosporin, against pathogens collected from dogs and cats in Europe and North America. Antimicrobial Agents and Chemotherapy, Washington, v. 50, n. 7, p. 2286-2292, 2006a.

STEGEMANN, M. R.; SHERINGTON, J.; COATI, N.; BROWN, S. A.; BLANCHFLOWER, S. Pharmacokinetics of cefovecin in cats. Journal of Veterinary Pharmacolology and Therapeutics, Oxford, v. 29 , n. 6 , p. $513-524,2006$ b.
STEGEMANN, M. R.; SHERINGTON, J.; BLANCHFLOWER, S. Pharmacokinetics and pharmacodynamics of cefovecin in dogs. Journal of Veterinary Pharmacolology and Therapeutics, Oxford, v. 29, n. 6, p. 501-511, 2006.

TRAŞ, B.; MADEN, M.; BAŞ, A. L.; ELMAS, M.; YAZAR, E.; CIVELEK, T. Investigation of biochemical and haematological side-effects of enrofloxacin in dogs. Journal of Veterinary Medicine. A, Physiology, Pathology, Clinical Medicine, Berlin, v. 48, n. 1, p. 5963, 2001.

VADEN, S. L.; RIVIERE, J. E. Penicilinas e antibióticos betalactâmicos. In.: ADAMS, H. R. (Ed.). Farmacologia e terapêutica em veterinária. Rio de Janeiro: Guanabara Koogan, 2003. 683 p.

WERNICK, M. B.; MÜNTENER, C. R. Cefovecin: a new long-acting cephalosporin. Journal of Exotic Pet Medicine, New York, v. 19, n. 4, p. 317-322, 2010. 
